\title{
Combining Ability in Ridge Gourd [Luffa acutangula]
}

\author{
Divya S. Kamble*, Shivaji Duradundi V.D. Gasti, Shashikanth Evoor, \\ Dileep Kumar A. Masuthi and Sumangala Koulagi
}

\author{
Department of Vegetable Science, Kittur Rani Channamma College of Horticulture, \\ Arabhavi - 591 218, Karnataka, India \\ *Corresponding author
}

\section{A B S T R A C T}

\begin{tabular}{|c|c|}
\hline Key w o r ds & Line $\mathrm{x}$ Tester analysis was carried out for ten diversified parents at the De \\
\hline $\begin{array}{l}\text { Combining ability, } \\
\text { Variance, General } \\
\text { combinig ability, } \\
\text { Specific combining } \\
\text { abiliy }\end{array}$ & $\begin{array}{l}\text { combining ability for various characters in ridge gourd. Lines DWD local, ERG-2, ERG-1 } \\
\text { and DMRG-1 were identified as good general combiners for yield appear to be worthy of } \\
\text { exploitation in future hybrid development. It is suggested that population involving these } \\
\text { lines may be developed through multiple crossing isolating high yielding varieties. The }\end{array}$ \\
\hline Article Info & GCA to SCA ratio for various characters indicate that the presence of both additive and \\
\hline $\begin{array}{l}\text { Accepted: } \\
07 \text { November } 2018 \\
\text { Available Online: } \\
\text { 10 December } 2018\end{array}$ & $\begin{array}{l}\text { Deepthi, HSN local x CO-1 and DMRG- } 25 \times \text { Deepthi were identified as good specific } \\
\text { combiners for total fruit yield per hectare in order of merit. These cross combinations can } \\
\text { be utilized for further breeding programme for crop improvement in ridge gourd. }\end{array}$ \\
\hline
\end{tabular}

\section{Introduction}

Ridge or ribbed gourd (Luffa acutangula Roxb., $2 \mathrm{n}=2 \mathrm{x}=26$ ) is a popular cucurbitaceous vegetable grown as spring and summer season crop. The fruits have 10 prominent longitudinal ridges; thus the plant is also described as "angular loofah", "Chinese okra," ridged gourd," or "fluted loofah." Ridge gourd is one of the least expensive vegetables to produce. It is cultivated on commercial scale and in kitchen garden.

Combining ability analysis has greater importance in crop improvement to identification of best combiners and utilize them in hybridization programme to produce superior hybrids, either to exploit for heterosis or to combine favourable genes (Meena et al., 2015). This technique was developed by Kempthorne in 1957. In addition, the information on nature of gene action will be helpful to develop efficient crop improvement programme. General combining ability is due to additive and additive $x$ additive gene action and is fixable in nature while specific combining ability is due to non-additive gene action which may be due to dominance or epistasis or both and is non-fixable. The presence of non-additive genetic variance is 
the primary justification for initiating the hybrid breeding programme (Pali and Mehta, 2014). Keeping these points in view, the present investigation was undertaken to determine general combining ability and specific combining ability in ridge gourd.

\section{Materials and Methods}

The parental materials developed at the Department of Vegetable Science, Kittur Rani Channamma College of Horticulture, Arabhavi were utilized for the study. The lines used in the present investigation were collected from local areas of Arabhavi region, Hassan region, Chikkamagaluru region and Dharwad region. Subsequently these lines were selected based on their per se performance for yield and quality attributes. Testers used were CO-1, Deepthi and Pusa Nutan, selected on basis of their earliness and high yielding character.

The experimental plot was ploughed repeatedly and land was brought to a fine tilth. About 20 tonnes of FYM per hectare and the recommended basal dose of fertilizers (25:50:50 kg NPK) were incorporated into the soil just before sowing. The remaining $25 \mathrm{~kg}$ of nitrogen was applied as a top dress at 45 days after sowing. Ridges and furrows were opened at a distance of $1.20 \mathrm{~m}$ apart. Two seeds of each genotype per hill were dibbled at a distance of $0.90 \mathrm{~m}$ in a row. Irrigation, weed control and other cultural practices were followed as per the package of practices of UHS, Bagalkot (Anon., 2014a).

\section{Results and Discussion}

Highly significant gca effects was reported for vine length at 45 DAS in both positive and negative direction. Significant positive gca effects was found in HSN local (46.67) followed by IC-92637 (23.00) among lines. Two lines also showed significant positive gca effects. PSN showed highly significant positive gca effects (5.12) among testers where other two showed negative gca effects. Among crosses sca effect was recorded in both negative and positive direction, where eight crosses showed highly significant positive sca effects for vine length at 45 DAS. Among 30 crosses highest sca effects was found in ERG-1 x Deepthi (81.39) followed by DMRG-3 x PSN (75.73). For vine length at 90 DAS, six lines showed highly significant positive gca effects, among which IC-92637 showed highest gca effects (31.44) followed by ERG-1 (27.77). Only three lines showed negative gca effects. Among testers only Deepthi showed highly significant positive gca effects and other (17.93) two testers were significant in negative direction. Among crosses, 10 crosses were reported to be highly significant in positive direction and cross DMRG-3 x PSN showed highest positive sca effects (41.21) followed by CKM local x PSN (32.88).Highly significant gca effects was reported for number of leaves at 45 DAS in both positive and negative direction. Highly significant positive gca effects was found in DWD local (13.09) followed by DMRG-1 (8.32) among lines. One line also showed significant positive gca effects among 10 lines. CO-1 showed highly significant positive gca effects (4.09) among testers where other two did not show significant gca effects. Among crosses sca effects was recorded in both negative and positive direction, where only a cross DWD local x CO-1 was reported to have highly significant positive sca effects (12.51) for number of leaves at 45 DAS followed by DMRG-1 x CO-1 (9.38).

For number of leaves at 90 DAS, IC-92637 showed highest gca effects (6.31) followed by DMRG-3 (6.25). None of the testers showed significant gca effects. Among crosses, ERG-1 $x$ Deepthi showed highest positive sca effects (5.54) followed by DWD local $x$ PSN (4.92).Highly significant gca effects was 
reported for number of branches at 45 DAS in both positive and negative direction. Highly significant positive gca effects was found in only DWD local (1.18) among 10 lines. None of the testers showed significant positive gca effects. Among crosses only DWD local $\mathrm{x}$ PSN was reported to have highly significant positive sca effects (1.09) for number of branches at 45 DAS. For number of branches at 90 DAS, DMRG-3 (1.16) showed highest gca effects followed by IC-92637 (0.96). Among testers PSN showed significant positive gca effects (0.26). Among crosses, ERG-2 x PSN showed highest positive sca effects (0.97) followed by DMRG-1 x Deepthi (0.90).

For days to first male flowering only DMRG25 (-2.18) showed highest significant negative gca effects as gca effects in negative direction is considered to be desirable for earliness parameters. None of the testers showed significant negative gca effects. Among 30 crosses, only cross DWD local x PSN showed highly significant sca effects (-6.55) in negative direction followed by the cross ERG$4 \mathrm{x}$ PSN (-4.23).For node to first male flowering only DMRG-25 (-0.42) showed highest significant negative gca effects as gca effects in negative direction is considered to be desirable for earliness parameters. None of the testers showed significant negative gca effects. Among 30 crosses, maximum and significant sca effects in negative direction was reported in cross ERG-4 x CO-1 (-1.08) followed by ERG-4 x Deepthi (-0.89) and DWD local $x$ PSN (-0.56).For days to first female flowering none of the lines and testers showed significant negative gca effects. Among 30 crosses, only cross DMRG-1 x Deepthi showed highly significant sca effects $(-7.05)$ in negative direction. Only two crosses showed significant sca effects in positive direction. For node to first female flowering only HSN local (-1.56) showed highest significant negative gca effects as gca effects in negative direction. Whereas, DWD local showed highest significant positive gca effects. Only CO-1 (-0.94) showed significant negative gca effects among testers. Among 30 crosses, maximum and significant sca effects in negative direction were reported in cross DWD local x CO-1 (-3.26). None of the other crosses showed significant negative sca effects.

For days to first harvest, among 10 lines, CKM local was reported to have highest significant negative gca effects (-7.65) followed by IC-92637 (-4.80). None of testers showed significant negative gca effects. Among the crosses, DWD local x CO-1 (9.71) followed by ERG-4 x Deepthi (-9.70), DMRG-1 x CO-1 (-8.81) exhibited highest significant negative sca effects for days to first harvest. Among 10 lines, IC-92637 was reported to have highest significant negative gca effects (-3.83) for days to last harvest. None other lines showed significant negative gca effects. None of tester showed significant negative gca effects. None of the crosses exhibited significant negative sca effects for days to last harvest. For sex ratio, ERG-2 showed highly significant negative gca effects (-2.26). None of tester showed significant negative gca effects. Out of 30 crosses, none of the crosses showed significant negative sca effects for sex ratio.

For fruit length, DMRG-25 (4.61) showed highest significant positive gca effects followed by DMRG-3 (3.25). Two other lines also reported highest significant positive gca effects among 10 lines. None of the tester showed significant positive gca effects. Among 30 crosses, ERG-1 x PSN (5.26) followed by DMRG-25 x CO-1 (5.08) exhibited highest positive sca effects for fruit length. Two other crosses also showed highest positive sca effects for fruit length. For fruit diameter, CKM local (0.59) showed highest significant positive gca effects followed by 
DWD local (0.48) and IC-92637 (0.43) among 10 lines. Two of the 10 lines showed significant negative gca effects. Only CO-1 showed significant positive gca effects (0.29) among testers. Among 30 crosses, HSN local x CO-1 (1.16) followed by ERG-4 x Deepthi (0.89) exhibited highest positive sca effects for fruit diameter. Two other crosses also showed highest positive sca effects.

For percent fruit set, none of the lines showed significant positive gca effects. None other lines showed significant positive gca effects. Among testers CO-1 (3.52) followed by PSN (3.24) showed highly significant positive gca effects. Out of 30 crosses, only DMRG-3 x Deepthi (15.26) showed significant positive sca effects followed by ERG-1 x CO- 1 (12.25).

Highly significant gca effects was reported for average fruit weight in both positive and negative direction. Highly significant positive gca effects was found in DWD local (29.65) followed by DMRG-25 (14.33) among lines. Deepthi (8.91) showed highly significant positive gca effects among testers where other two did not show significant positive gca effects. Among crosses sca effects was recorded in both negative and positive direction, where only a cross ERG-4 x CO-1 was reported to have highly significant positive sca effects (46.88) followed by ERG2 x Deepthi (42.19) and DWD local x CO-1 (23.23) for averege fruit weight.

For number of fruits per vine, ERG-2 (1.80) showed significant positive gca effects followed by DWD local (1.37) among 10 lines. None of tester showed significant positive gca effects. Out of 30 crosses, ERG-4 $x$ Deepthi showed significant positive sca effects (2.04) followed by DMRG-3 x Deepthi (1.80) for number of fruits per vine. Highly significant gca effects was reported for fruit yield per vine in both positive and negative direction. Highly significant positive gca effects was found in ERG-2 (0.39) followed by DWD local (0.34) among lines. Two lines also showed significant negative gca effects among 10 lines. Deepthi (0.07) showed highly significant positive gca effects among testers and other two did not show significant positive gca effects. Among crosses sca effects was recorded in both negative and positive direction and the cross DWD local $\mathrm{x}$ CO-1 was reported to have highly significant positive sca effects $(0.88)$ followed by ERG-2 x Deepthi (0.65).

Highly significant gca effects was reported for fruit yield per plot in both positive and negative direction. Highly significant positive gca effects was found in ERG-2 (3.53) followed by DWD local (2.62) and ERG-1 (2.35) among lines. None of the testers showed positive gca effects. Among crosses sca effects was recorded in both negative and positive direction, where only a cross DWD local x CO-1 was reported to have highly significant positive sca effects (3.92) followed by ERG-1 x Deepthi (2.22), IC-92637 x Deepthi (2.09) and HSN local x CO-1 (1.95) for fruit yield per plot.

Highly significant gca effects was reported for yield per hectare in both positive and negative direction. High significant positive gca effects was found in ERG-2 (32.72) followed by DWD local (24.23) and ERG-1 (21.76) among lines. Deepthi (7.5) showed highly significant positive gca effects among testers where PSN (-8.44) showed significant negative gca effects. Among crosses sca effects was recorded in both negative and positive direction, where only a cross DWD local $\mathrm{x}$ CO-1 was reported to have highly significant positive sca effects (36.28) followed by ERG1 x Deepthi (20.56) and IC-92637 x Deepthi (19.32). Two other crosses also exhibited significant positive sca effects for fruit yield per hectare. 
None of the lines showed significant positive gca effects for rind thickness, but $\mathrm{CO}-1$ showed significant positive gca effects $(0.37)$ among three testers. Out of 30 crosses, only the cross CKM local x Deepthi showed highly significant sca effects (1.55) in positive direction for rind thickness.For flesh thickness, ERG-1 showed significant positive gca effects (0.78) followed by HSN local (0.61). Deepthi showed significant positive gca effects (0.46) among three testers. Out of 30 crosses, only the cross ERG-4 x PSN showed highly significant sca effects (1.5) in positive direction. None other crosses showed significant positive sca effects for flesh thickness (Table 1-3).

Variance for all 23 characters revealed that non additive gene action was predominant for days to last harvest and days to first female flowering these traits can be improved by recurrent selection of breeding method where as non additive component was more than additive component for vine length at 45 DAS and 90 DAS, number of branches at 45 and 90 DAS, number of leaves at 45 and 90 DAS, days to first male flowering, node to first male flowering, days to first harvest, days to last harvest, sex ratio, fruit length, fruit diameter, number of fruits per vine, fruit yield per vine, fruit yield per plot, fruit yield per hectare, per cent fruit set, flesh thickness and rind thickness. These traits can be improved by recurrent selection. But for average fruit weight and node to first female flowering additive components of genetic variance were predominant and thus can be improved by simple selection or direct selection.

Table.1 Details of ridge gourd entries with their sources

\begin{tabular}{|c|c|c|c|}
\hline SI. No. & Entry & Pedigree/Name & Source \\
\hline \multicolumn{4}{|l|}{ Lines } \\
\hline 1. & L- 1 & IC-92637 & KRCCH, Arabhavi \\
\hline 2 & L- 2 & DMRG-3 & KRCCH, Arabhavi \\
\hline 3. & L- 3 & DMRG-25 & KRCCH, Arabhavi \\
\hline 4. & L- 4 & Chikkamagaluru local & Chikkkamagaluru \\
\hline 5. & L- 5 & ERG-4 & KRCCH, Arabhavi \\
\hline 6. & L- 6 & Hassan local & Belur, Hassan \\
\hline 7. & L- 7 & DMRG-1 & KRCCH, Arabhavi \\
\hline 8. & L- 8 & ERG-1 & KRCCH, Arabhavi \\
\hline 9. & L- 9 & Dharwad local & Dharwad \\
\hline 10. & L- 10 & ERG-2 & KRCCH, Arabhavi \\
\hline \multicolumn{4}{|l|}{ Testers } \\
\hline 1. & Deepthi & & KAU, Kerala \\
\hline 2. & PSN & Pusa Nutan & IARI, New delhi \\
\hline 3. & $\mathrm{CO}-1$ & & TNAU, Coimbotore \\
\hline \multicolumn{4}{|c|}{ Commercial check } \\
\hline 1. & Naga & $F_{1}$ hybrid & East-West company \\
\hline
\end{tabular}


Table.2 General combining ability effects for growth, earliness, yield and quality parameters in ridge gourd

\begin{tabular}{|c|c|c|c|c|c|c|c|c|c|c|c|c|c|c|}
\hline \multirow[t]{2}{*}{ Sl. No. } & \multirow[t]{2}{*}{ Parents } & \multicolumn{2}{|c|}{ Vine length } & \multicolumn{2}{|c|}{ Number of leaves } & \multicolumn{2}{|c|}{$\begin{array}{l}\text { Number of } \\
\text { branches }\end{array}$} & \multirow{2}{*}{$\begin{array}{c}\text { Days to } \\
\text { first } \\
\text { male } \\
\text { flowering }\end{array}$} & \multirow{2}{*}{$\begin{array}{c}\text { Node to } \\
\text { first } \\
\text { male } \\
\text { flowering }\end{array}$} & \multirow{2}{*}{$\begin{array}{c}\text { Days to } \\
\text { first } \\
\text { female } \\
\text { flowering }\end{array}$} & \multirow{2}{*}{$\begin{array}{c}\text { Node to } \\
\text { first } \\
\text { female } \\
\text { flowering }\end{array}$} & \multirow{2}{*}{$\begin{array}{c}\text { Days } \\
\text { to first } \\
\text { harvest }\end{array}$} & \multirow{2}{*}{$\begin{array}{c}\text { Days } \\
\text { to last } \\
\text { harvest }\end{array}$} & \multirow{2}{*}{$\begin{array}{c}\text { Sex } \\
\text { ratio }\end{array}$} \\
\hline & & 45 DAS & 90 DAS & 45 DAS & 90 DAS & 45 DAS & 90 DAS & & & & & & & \\
\hline \multicolumn{15}{|c|}{ Lines } \\
\hline 1 & IC-92637 & $23.00 * *$ & $31.44 * *$ & -4.51 & $6.31 * *$ & 0.03 & $0.96 * *$ & 0.69 & -0.09 & 2.69 & -0.5 & $-4.80 * *$ & $-3.83^{*}$ & -0.02 \\
\hline 2 & DMRG-3 & $16.67 * *$ & $16.92 * *$ & -4.84 & $6.25 * *$ & -0.34 & $1.16 * *$ & 0.13 & -0.06 & -1.58 & -1.1 & $4.10 * *$ & -2.83 & $2.58 * *$ \\
\hline 3 & DMRG-25 & 0.87 & $21.64 * *$ & $-7.94 * *$ & -1.35 & -0.21 & $-1.04 * *$ & $-2.18 *$ & $-0.42 *$ & -1.11 & -0.73 & $4.26 * *$ & 0.33 & -0.49 \\
\hline 4 & CKM local & $14.50 * *$ & $22.04 * *$ & $-5.38 *$ & -1.19 & -0.08 & -0.21 & 0.09 & $-0.32 *$ & 1.54 & 0.4 & $-7.65 * *$ & 0.33 & 0.03 \\
\hline 5 & ERG-4 & $-33.33 * *$ & $-40.93 * *$ & $-9.34 * *$ & $-3.62 *$ & $-0.58 *$ & $-1.11 * *$ & -1.84 & $0.81 * *$ & -2.06 & -1.13 & $-2.62 *$ & 2.33 & -0.56 \\
\hline 6 & HSN local & $46.67 * *$ & $-58.68 * *$ & -0.64 & 1.65 & -0.38 & $0.39 *$ & 0.19 & -0.29 & 1.92 & $-1.56^{*}$ & $4.73 * *$ & 2.67 & 0.28 \\
\hline 7 & DMRG-1 & $-10.33 * *$ & $12.50 * *$ & $8.32 * *$ & $-3.22 *$ & -0.14 & -0.14 & -1.31 & -0.19 & -0.66 & -0.4 & -0.44 & 2.67 & 0.52 \\
\hline 8 & ERG-1 & $-20.13 * *$ & $27.77 * *$ & 4.26 & -1.75 & 0.26 & $0.49 * *$ & 0.09 & 0.31 & 0.42 & -0.4 & 0.73 & $3.67 *$ & 0.85 \\
\hline 9 & DWD local & $-17.46 * *$ & -2.53 & $13.09 * *$ & $-4.09 * *$ & $1.18 * *$ & -0.24 & $3.07 * *$ & 0.04 & -1.34 & $4.27 * *$ & -1.34 & -2 & -0.93 \\
\hline \multirow[t]{5}{*}{10} & ERG-2 & $-20.48 * *$ & $-30.16 * *$ & $6.99 *$ & 1.01 & 0.26 & -0.24 & 1.06 & 0.21 & 0.17 & 1.14 & 2.13 & -3.33 & $-2.26 * *$ \\
\hline & SE.m \pm & 1.75 & 1.19 & 1.81 & 0.96 & 0.16 & 0.11 & 0.74 & 0.11 & 0.93 & 0.41 & 0.82 & 1.23 & 0.53 \\
\hline & CD at $5 \%$ & 5.07 & 4.46 & 5.24 & 2.79 & 0.45 & 0.34 & 2.14 & 0.32 & 2.8 & 1.2 & 2.37 & 3.5 & 1.54 \\
\hline & CD at $1 \%$ & 6.83 & 6.01 & 7.06 & 3.75 & 0.61 & 0.45 & 2.88 & 0.44 & 3.72 & 1.62 & 3.2 & 4.68 & 2.07 \\
\hline & Testers & & & & & & & & & & & & & \\
\hline 1 & Deepthi & $-3.97 * *$ & $17.93 * *$ & -1.92 & -0.21 & $-0.38 * *$ & $-0.37 * *$ & 1.04 & -0.14 & -0.34 & 0.39 & -1.03 & 0.28 & 0.35 \\
\hline 2 & PSN & $5.12 * *$ & $-13.31 * *$ & -2.17 & -0.85 & 0.24 & $0.26 * *$ & -0.97 & $0.30 * *$ & -0.66 & 0.55 & 1.09 & 0.28 & -0.4 \\
\hline \multirow[t]{5}{*}{3} & $\mathrm{CO}-1$ & $-1.16 * *$ & $-4.61 * *$ & $4.09 * *$ & 1.06 & 0.13 & 0.11 & -0.07 & -0.15 & 1 & $-0.94 * *$ & -0.05 & -0.57 & 0.06 \\
\hline & SE.m \pm & 0.96 & 0.84 & 0.99 & 0.53 & 0.09 & 0.06 & 0.4 & 0.6 & 0.51 & 0.23 & 0.45 & 0.67 & 0.29 \\
\hline & CD at $5 \%$ & 2.77 & 2.44 & 2.87 & 1.53 & 0.25 & 0.18 & 1.17 & 0.18 & 1.54 & 0.66 & 1.3 & 1.92 & 0.84 \\
\hline & CD at $1 \%$ & 3.74 & 3.3 & 3.87 & 2.06 & 0.33 & 0.25 & 1.58 & 0.24 & 2.04 & 0.88 & 1.75 & 2.56 & 1.14 \\
\hline & & & & & & & & 1.04 & -0.14 & -0.34 & 0.39 & -1.03 & 0.28 & 0.35 \\
\hline
\end{tabular}

*and $* *$ indicate significance of values at $\mathrm{p}=0.05$ and $\mathrm{p}=0.01$, respectively.

DAS: Days after sowing 
Table. 2 contd...

\begin{tabular}{|c|c|c|c|c|c|c|c|c|c|}
\hline $\begin{array}{l}\text { Sl. } \\
\text { No. }\end{array}$ & Parents & Fruit length & Fruit diameter & Per cent fruit set & Averege fruit weight & $\begin{array}{l}\text { Number of } \\
\text { fruits per vine }\end{array}$ & $\begin{array}{l}\text { Fruit yield } \\
\text { per vine }\end{array}$ & $\begin{array}{c}\text { Fruit yield } \\
\text { per plot }\end{array}$ & $\begin{array}{c}\text { Fruit yield } \\
\text { per }\end{array}$ \\
\hline & & & & & & & & & \\
\hline \multicolumn{10}{|c|}{ Lines } \\
\hline 1 & IC-92637 & $2.34 * *$ & $0.43 *$ & -4.72 & $2.34 * *$ & $0.43 *$ & -4.72 & $-1.11 *$ & $-10.34 *$ \\
\hline 2 & DMRG-3 & $3.25 * *$ & 0.29 & $-11.23 * *$ & $3.25 * *$ & 0.29 & $-11.23 * *$ & $-1.13 *$ & $-10.50 *$ \\
\hline 3 & DMRG-25 & $4.61 * *$ & -0.08 & 2.11 & $4.61 * *$ & -0.08 & 2.11 & $-1.12 *$ & $-10.34^{*}$ \\
\hline 4 & CKM local & -1.21 & $0.59 * *$ & 7.30 & -1.21 & $0.59 * *$ & 7.30 & -0.95 & -8.8 \\
\hline 5 & ERG-4 & $-2.30 * *$ & -0.21 & -9.57 & $-2.30 * *$ & -0.21 & -9.57 & -0.68 & -6.33 \\
\hline 6 & HSN local & $2.90 * *$ & $-0.96 * *$ & 5.83 & $2.90 * *$ & $-0.96 * *$ & 5.83 & $-2.02 * *$ & $-18.67 * *$ \\
\hline 7 & DMRG-1 & $-3.08 * *$ & -0.15 & 8.78 & $-3.08 * *$ & -0.15 & 8.78 & $-1.48 * *$ & $-13.73 * *$ \\
\hline 8 & ERG-1 & $-2.60 * *$ & $-0.55 * *$ & -4.34 & $-2.60 * *$ & $-0.55 * *$ & -4.34 & $2.35 * *$ & $21.76^{* *}$ \\
\hline 9 & DWD local & $-2.95 * *$ & $0.48 * *$ & 8.31 & $-2.95 * *$ & $0.48 * *$ & 8.31 & $2.62 * *$ & $24.23 * *$ \\
\hline \multirow[t]{5}{*}{10} & ERG-2 & -0.97 & 0.17 & -2.45 & -0.97 & 0.17 & -2.45 & $3.53 * *$ & $32.72 * *$ \\
\hline & SE.m \pm & 0.50 & 0.11 & 1.94 & 0.50 & 0.11 & 1.94 & 0.33 & 3.04 \\
\hline & CD at $5 \%$ & 1.43 & 0.33 & 5.82 & 1.43 & 0.33 & 5.82 & 0.95 & 8.81 \\
\hline & CD at $1 \%$ & 1.93 & 0.44 & 7.76 & 1.93 & 0.44 & 7.76 & 1.28 & 11.87 \\
\hline & Testers & & & & & & & & \\
\hline 1 & Deepthi & 0.20 & -0.17 & $-6.76 * *$ & 0.20 & -0.17 & $-6.76 * *$ & 0.07 & $7.5 * *$ \\
\hline 2 & PSN & 0.08 & -0.12 & $3.24 *$ & 0.08 & -0.12 & $3.24 *$ & -0.11 & $-8.44 * *$ \\
\hline \multirow[t]{5}{*}{3} & $\mathrm{CO}-1$ & -0.28 & $0.29 * *$ & $3.52 *$ & -0.28 & $0.29 * *$ & $3.52 *$ & 0.04 & 0.91 \\
\hline & SE.m \pm & 0.27 & 0.06 & 1.06 & 0.27 & 0.06 & 1.06 & 0.18 & 1.67 \\
\hline & CD at $5 \%$ & 0.79 & 0.18 & 3.19 & 0.79 & 0.18 & 3.19 & 0.52 & 4.82 \\
\hline & CD at $1 \%$ & 1.10 & 0.24 & 4.25 & 1.10 & 0.24 & 4.25 & 0.70 & 6.49 \\
\hline & & $2.34 * *$ & $0.43 *$ & -4.72 & $2.34 * *$ & $0.43 *$ & -4.72 & $-1.11 *$ & $-10.34 *$ \\
\hline
\end{tabular}

*and **indicate significance of values at $\mathrm{p}=0.05$ and $\mathrm{p}=0.01$, respectively. 
Table.3 Specific combining ability for growth, earliness, yeild and quality parameters in ridge gourd

\begin{tabular}{|c|c|c|c|c|c|c|c|c|c|c|c|c|c|}
\hline \multirow[t]{2}{*}{$\begin{array}{l}\text { Sl. } \\
\text { No. }\end{array}$} & \multirow[t]{2}{*}{ Hybrids } & \multicolumn{2}{|c|}{ Vine length } & \multicolumn{2}{|c|}{ Number of leaves } & \multicolumn{2}{|c|}{$\begin{array}{l}\text { Number of } \\
\text { branches }\end{array}$} & \multirow{2}{*}{$\begin{array}{l}\text { Days to } \\
\text { first male } \\
\text { flowering }\end{array}$} & \multirow{2}{*}{$\begin{array}{l}\text { Node to } \\
\text { first male } \\
\text { flowering }\end{array}$} & \multirow{2}{*}{$\begin{array}{l}\text { Days to } \\
\text { first } \\
\text { female } \\
\text { flowering }\end{array}$} & \multirow{2}{*}{$\begin{array}{l}\text { Node to } \\
\text { first } \\
\text { female } \\
\text { flowering }\end{array}$} & \multirow{2}{*}{$\begin{array}{l}\text { Days to } \\
\text { first } \\
\text { harvest }\end{array}$} & \multirow{2}{*}{$\begin{array}{l}\text { Days to } \\
\text { last } \\
\text { harvest }\end{array}$} \\
\hline & & 45 DAS & 90 DAS & 45 DAS & 90 DAS & 45 DAS & 90 DAS & & & & & & \\
\hline 1 & IC-92637 x Deepthi & -2.53 & -2.94 & 0.52 & 2.68 & 0.01 & 0.20 & -1.28 & -0.09 & 2.81 & 0.58 & -3.77 & -1.12 \\
\hline 2 & IC-92637 x PSN & -0.29 & -6.12 & 4.17 & -4.08 & 0.29 & -0.13 & 0.94 & -0.23 & -0.77 & 0.52 & $-4.89 *$ & 0.88 \\
\hline 3 & IC-92637 x CO-1 & 2.81 & $9.06^{*}$ & -4.69 & 1.41 & -0.3 & -0.08 & 0.34 & 0.32 & -2.04 & -1.09 & $8.65 * *$ & 0.23 \\
\hline 4 & DMRG-3 x Deepthi & $-25.26^{* *}$ & -3.20 & 2.05 & 1.94 & 0.28 & 0.00 & -0.51 & 0.18 & 3.37 & -1.82 & 3.23 & 1.38 \\
\hline 5 & DMRG-3 x PSN & $75.73 * *$ & $41.21 * *$ & 5.20 & 2.08 & 0.06 & $0.67 *$ & 1.71 & -0.36 & -2.90 & 0.72 & $-4.29 *$ & -0.12 \\
\hline 6 & DMRG-3 x CO-1 & $-50.47 * *$ & $-38.01 * *$ & -7.26 & -4.03 & -0.33 & $-0.68 *$ & -1.20 & 0.19 & -0.47 & 1.11 & 1.05 & -1.27 \\
\hline 7 & DMRG-25 x Deepthi & $-41.73^{* *}$ & -4.80 & 2.15 & -0.76 & 0.04 & 0.30 & -0.71 & 0.04 & -0.05 & -0.49 & -0.43 & -2.28 \\
\hline 8 & DMRG-25 x PSN & $10.01 *$ & -6.99 & 1.10 & 0.28 & -0.38 & -0.53 & -2.10 & -0.30 & 0.38 & 0.45 & -1.75 & -0.28 \\
\hline 9 & DMRG-25 x CO-1 & $31.71 * *$ & $11.79 * *$ & -3.26 & 0.47 & 0.33 & 0.22 & 2.81 & 0.25 & -0.34 & 0.04 & 2.19 & 2.57 \\
\hline 10 & CKM local x Deepthi & $-14.13^{* *}$ & $-31.04 * *$ & 2.39 & -3.32 & 0.51 & 0.27 & -2.18 & 0.24 & 0.26 & 1.08 & -0.92 & 0.22 \\
\hline 11 & CKM local x PSN & 5.11 & $32.88 * *$ & 2.54 & 0.82 & -0.21 & -0.56 & 1.14 & 0.20 & 0.23 & -1.28 & -0.39 & -0.78 \\
\hline 12 & CKM local x CO-1 & $9.01^{*}$ & -1.85 & -4.92 & 2.51 & -0.30 & 0.29 & 1.04 & -0.45 & -0.49 & 0.21 & 1.30 & 0.57 \\
\hline 13 & ERG-4 x Deepthi & $-18.16^{* *}$ & 4.80 & -2.25 & -3.59 & 0.01 & 0.37 & 1.86 & $-0.89 * *$ & 0.61 & 0.71 & $9.35 * *$ & -0.28 \\
\hline 14 & ERG-4 x PSN & $31.28 * *$ & $-27.79 * *$ & 0.80 & 1.25 & -0.51 & $-1.16^{* *}$ & $-4.23 *$ & $1.97 * *$ & 0.28 & -0.75 & $-5.82 * *$ & -1.28 \\
\hline 15 & ERG-4 x CO-1 & $-13.12 * *$ & $22.99 * *$ & 1.44 & 2.34 & 0.5 & $0.79 * *$ & 2.37 & $-1.08 * *$ & -0.89 & 0.04 & -3.53 & 1.57 \\
\hline 16 & HSN local x Deepthi & $-24.41^{* *}$ & $18.80 * *$ & 5.75 & -1.16 & 0.31 & $-0.83 * *$ & -0.88 & -0.09 & -2.13 & -0.56 & 2.50 & 2.88 \\
\hline 17 & HSN local x PSN & $26.78 * *$ & $-28.29 * *$ & 0.10 & -0.72 & -0.31 & 0.54 & 1.94 & -0.23 & -4.30 & 0.48 & -1.72 & -1.12 \\
\hline 18 & HSN local x CO-1 & -2.37 & $9.49 *$ & -5.86 & 1.87 & 0.00 & 0.29 & -1.06 & 0.32 & $6.43 *$ & 0.07 & -0.78 & -1.77 \\
\hline 19 & DMRG-1 x Deepthi & $-14.59 * *$ & 6.80 & $-11.01 *$ & 3.61 & 0.28 & $0.90 * *$ & -0.88 & 0.31 & $-7.05 * *$ & -1.02 & 2.97 & -1.62 \\
\hline 20 & DMRG-1 x PSN & -5.35 & -0.79 & 1.64 & $-6.45 *$ & -0.04 & $-0.73 *$ & 1.14 & -0.03 & $5.18^{*}$ & 0.02 & $5.85 * *$ & 0.38 \\
\hline 21 & DMRG-1 x CO-1 & $19.95 * *$ & -6.01 & $9.38 *$ & 2.84 & -0.23 & -0.18 & -0.26 & -0.28 & 1.87 & 1.01 & $-8.81 * *$ & 1.23 \\
\hline 22 & ERG-1 x Deepthi & $81.39 * *$ & $13.60 * *$ & 4.95 & $5.54 *$ & -0.62 & -0.03 & -0.68 & 0.01 & -1.53 & -0.62 & $-6.90 * *$ & 3.38 \\
\hline 23 & ERG-1 x PSN & $-81.12 * *$ & $-38.89 * *$ & -5.1 & 0.58 & 0.56 & 0.54 & 2.24 & -0.13 & 1.20 & -0.58 & 1.28 & 0.38 \\
\hline 24 & ERG-1 x CO-1 & -0.27 & $25.29 * *$ & 0.14 & $-6.13 *$ & 0.07 & -0.51 & -1.56 & 0.12 & 0.33 & 1.21 & $5.62 * *$ & -3.77 \\
\hline 25 & DWD local x Deepthi & -8.16 & $18.03 * *$ & 3.42 & -4.52 & $-0.79 *$ & -0.50 & $6.09 * *$ & 0.48 & 2.44 & 1.71 & 3.67 & -0.95 \\
\hline 26 & DWD local x PSN & $-32.38 * *$ & $15.85^{* *}$ & $-15.93 * *$ & $4.92 *$ & $1.09 * *$ & 0.37 & $-6.55 * *$ & $-0.56 *$ & 1.76 & 1.55 & $6.05 * *$ & -0.45 \\
\hline 27 & DWD local x CO-1 & $44.48 * *$ & $-33.88 * *$ & $12.51 * *$ & -0.39 & -0.3 & 0.12 & 0.46 & 0.09 & -4.20 & $-3.26 * *$ & $-9.71 * *$ & 1.40 \\
\hline 28 & ERG-2 $\times$ Deepthi & $67.57 * *$ & $-20.05^{* *}$ & -7.98 & -0.42 & -0.02 & $-0.70 *$ & -0.84 & -0.19 & 1.27 & 0.44 & $-9.70 * *$ & -1.62 \\
\hline
\end{tabular}


Int.J.Curr.Microbiol.App.Sci (2018) 7(12): 567-577

Table. 3 contd...

\begin{tabular}{|c|c|c|c|c|c|c|c|c|c|c|c|c|c|}
\hline \multirow[t]{2}{*}{$\begin{array}{l}\text { Sl. } \\
\text { No. }\end{array}$} & \multirow[t]{2}{*}{ Hybrids } & \multicolumn{2}{|c|}{ Vine length } & \multicolumn{2}{|c|}{ Number of leaves } & \multicolumn{2}{|c|}{$\begin{array}{l}\text { Number of } \\
\text { branches }\end{array}$} & \multirow{2}{*}{$\begin{array}{l}\text { Days to } \\
\text { first male } \\
\text { flowering }\end{array}$} & \multirow{2}{*}{$\begin{array}{l}\text { Node to } \\
\text { first male } \\
\text { flowering }\end{array}$} & \multirow{2}{*}{$\begin{array}{l}\text { Days to } \\
\text { first } \\
\text { female } \\
\text { flowering }\end{array}$} & \multirow{2}{*}{$\begin{array}{l}\text { Node to } \\
\text { first } \\
\text { female } \\
\text { flowering }\end{array}$} & \multirow{2}{*}{$\begin{array}{l}\text { Days to } \\
\text { first } \\
\text { harvest }\end{array}$} & \multirow{2}{*}{$\begin{array}{l}\text { Days to } \\
\text { last } \\
\text { harvest }\end{array}$} \\
\hline & & 45 DAS & 90 DAS & 45 DAS & 90 DAS & 45 DAS & 90 DAS & & & & & & \\
\hline 29 & ERG-2 x PSN & $-25.84 * *$ & $18.91 * *$ & 5.47 & 1.32 & -0.54 & $0.97 * *$ & $3.77 *$ & -0.33 & -1.05 & -1.12 & $5.68 * *$ & 2.38 \\
\hline 30 & ERG-2 x CO-1 & $-41.74 * *$ & 1.14 & 2.51 & -0.89 & 0.57 & -0.27 & -2.93 & 0.52 & -0.22 & 0.67 & 4.02 & -0.77 \\
\hline & SE.m \pm & 3.03 & 2.67 & 3.14 & 1.67 & 0.27 & 0.20 & 1.28 & 0.19 & 1.62 & 0.72 & 1.42 & 2.12 \\
\hline & CD at $5 \%$ & 8.78 & 7.73 & 9.07 & 4.82 & 0.78 & 0.58 & 3.70 & 0.56 & 4.88 & 2.08 & 4.11 & 6.06 \\
\hline & CD at $1 \%$ & 11.82 & 10.41 & 12.23 & 6.50 & 1.05 & 0.78 & 4.99 & 0.76 & 6.50 & 2.80 & 5.54 & 8.10 \\
\hline
\end{tabular}

*and **indicate significance of values at $\mathrm{p}=0.05$ and $\mathrm{p}=0.01$, respectively.

DAS: Days after sowing 
Table.3

\begin{tabular}{|c|c|c|c|c|c|c|c|c|c|c|c|c|}
\hline $\begin{array}{l}\text { Sl. } \\
\text { No. }\end{array}$ & Hybrids & Sex ratio & $\begin{array}{l}\text { Fruit } \\
\text { length }\end{array}$ & $\begin{array}{l}\text { Fruit } \\
\text { diameter }\end{array}$ & $\begin{array}{l}\text { Per cent } \\
\text { fruit set }\end{array}$ & $\begin{array}{l}\text { Average } \\
\text { fruit } \\
\text { weight }\end{array}$ & $\begin{array}{l}\text { Number of } \\
\text { fruits per } \\
\text { vine }\end{array}$ & $\begin{array}{l}\text { Fruit } \\
\text { yield per } \\
\text { vine }\end{array}$ & $\begin{array}{l}\text { Fruit } \\
\text { yield per } \\
\text { plot }\end{array}$ & $\begin{array}{l}\text { Fruit yield } \\
\text { per hectare }\end{array}$ & $\begin{array}{l}\text { Rind } \\
\text { thickness }\end{array}$ & $\begin{array}{l}\text { Flesh } \\
\text { thickness }\end{array}$ \\
\hline 1 & IC-92637 x Deepthi & 1.02 & -1.31 & 0.02 & -0.40 & 15.67 & 0.91 & -0.01 & $2.09 *$ & $19.32 *$ & -0.39 & 0.77 \\
\hline 2 & IC-92637 x PSN & -1.59 & -0.43 & 0.35 & 8.10 & 1.28 & -0.18 & 0.02 & -1.0 & -9.15 & -0.11 & -0.14 \\
\hline 3 & IC-92637 x CO-1 & 0.57 & 1.74 & -0.37 & -7.69 & -16.95 & -0.72 & -0.00 & -1.10 & -10.17 & 0.49 & -0.63 \\
\hline 4 & DMRG-3 x Deepthi & $2.71 *$ & -0.43 & -0.39 & $15.26^{* *}$ & -10.40 & $1.80^{*}$ & -0.03 & -0.70 & -6.45 & -0.39 & -0.96 \\
\hline 5 & DMRG-3 x PSN & -1.15 & 2.01 & 0.25 & $-10.90^{*}$ & 1.46 & -1.12 & 0.17 & 0.33 & 3.04 & 0.19 & 0.43 \\
\hline 6 & DMRG-3 x CO-1 & -1.56 & -1.58 & 0.14 & -4.36 & 8.93 & -0.66 & -0.14 & 0.37 & 3.41 & 0.19 & 0.54 \\
\hline 7 & DMRG-25 x Deepthi & 1.17 & -1.88 & 0.27 & -1.07 & -9.08 & 0.51 & -0.00 & $1.79 *$ & $16.54 *$ & -0.09 & 0.27 \\
\hline 9 & DMRG-25 x CO-1 & -1.98 & $5.08^{* *}$ & -0.33 & -5.53 & -1.45 & -0.52 & -0.06 & $-2.00 *$ & $-18.50 *$ & 0.09 & -0.23 \\
\hline 10 & CKM local x Deepthi & -0.60 & 1.73 & $0.59^{*}$ & $12.24 *$ & 0.85 & -0.73 & -0.10 & 0.62 & 5.74 & $1.55^{* *}$ & 0.70 \\
\hline 11 & CKM local x PSN & 1.26 & 1.52 & -0.01 & $-13.11^{*}$ & -11.46 & -0.12 & 0.09 & -1.06 & -9.77 & -0.57 & -1.01 \\
\hline 12 & CKM local x CO-1 & -0.67 & $-3.25 *$ & $-0.58 *$ & 0.87 & 10.61 & 0.84 & 0.01 & 0.44 & 4.03 & $-0.97 *$ & 0.30 \\
\hline 13 & ERG-4 x Deepthi & 0.33 & 1.51 & $0.89 * *$ & $10.27 *$ & $-31.75 * *$ & $2.04 *$ & -0.05 & $-2.05 *$ & $-18.95 *$ & -0.02 & $-1.40^{* *}$ \\
\hline 14 & ERG-4 x PSN & 0.98 & -1.29 & 0.43 & -2.59 & -15.14 & -0.85 & 0.10 & 0.73 & 6.74 & 0.06 & $1.5 * *$ \\
\hline 15 & ERG-4 x CO-1 & -1.32 & -0.21 & $-1.32 * *$ & -7.68 & $46.88 * *$ & -1.19 & -0.05 & 1.32 & 12.21 & -0.04 & -0.10 \\
\hline 16 & HSN local x Deepthi & -1.18 & $4.69 * *$ & $-0.61 *$ & -8.46 & 6.17 & -0.13 & 0.01 & $-2.41 * *$ & $-22.35 * *$ & -0.02 & 0.87 \\
\hline 17 & HSN local x PSN & 0.25 & $-5.04 * *$ & -0.55 & 5.37 & 16.68 & 0.68 & 0.02 & 0.46 & 4.27 & 0.06 & -0.74 \\
\hline 19 & DMRG-1 x Deepthi & 1.51 & $-4.30 * *$ & -0.15 & $-13.58 * *$ & 6.39 & -0.93 & -0.04 & 0.45 & 4.20 & 0.11 & 0.07 \\
\hline 20 & DMRG-1 x PSN & 0.55 & 1.142 & -0.55 & 7.44 & 12.25 & -0.72 & 0.05 & 0.28 & 2.58 & -0.41 & 0.26 \\
\hline 21 & DMRG-1 x CO-1 & -2.06 & $3.16 *$ & $0.70 *$ & 6.14 & -18.63 & 1.64 & -0.02 & -0.73 & -6.78 & 0.29 & -0.33 \\
\hline 22 & ERG-1 x Deepthi & -1.37 & $-2.93 *$ & -0.37 & -2.80 & 8.67 & -0.83 & 0.13 & $2.22 * *$ & $20.56 * *$ & -0.55 & -0.20 \\
\hline 23 & ERG-1 x PSN & 0.36 & $5.26 * *$ & 0.04 & -9.46 & -13.72 & 0.28 & -0.01 & -0.36 & -3.29 & 0.33 & -0.31 \\
\hline 24 & ERG-1 x CO-1 & 1.02 & -2.33 & 0.33 & $12.25^{*}$ & 5.05 & 0.54 & -0.12 & $-1.87 *$ & $-17.27 *$ & 0.23 & 0.50 \\
\hline 25 & DWD local x Deepthi & -1.34 & 1.62 & 0.04 & $-11.61^{*}$ & $-28.70 *$ & $-2.30 * *$ & $-0.60 * *$ & $-3.60^{* *}$ & $-33.30 * *$ & 0.31 & 0.20 \\
\hline 26 & DWD local x PSN & 0.04 & -0.57 & -0.27 & 6.90 & 5.46 & 1.12 & $-0.33 * *$ & -0.32 & -2.98 & 0.09 & 0.49 \\
\hline 27 & DWD local x CO-1 & 1.29 & -1.06 & 0.23 & 4.71 & $23.23 *$ & 1.18 & $0.88 * *$ & $3.92 * *$ & $36.28^{* *}$ & -0.41 & -0.70 \\
\hline 28 & ERG-2 x Deepthi & -2.26 & 1.29 & -0.28 & 0.15 & $42.19 * *$ & -0.33 & $0.65 * *$ & 1.59 & 14.69 & -0.52 & -0.33 \\
\hline 29 & ERG-2 x PSN & -1.51 & 0.60 & 0.24 & 1.65 & -7.35 & 0.88 & -0.18 & 0.71 & 6.59 & 0.36 & -0.44 \\
\hline \multirow[t]{4}{*}{30} & ERG-2 x CO-1 & $3.77 * *$ & -1.89 & 0.04 & -1.80 & $-34.83 * *$ & -0.56 & $-0.47 * *$ & $-2.29 * *$ & $-21.28 * *$ & 0.16 & 0.77 \\
\hline & SE.m \pm & 0.92 & 0.86 & 0.2 & 3.36 & 8.00 & 0.57 & 0.07 & 0.57 & 5.30 & 0.28 & 0.36 \\
\hline & CD at $5 \%$ & 2.67 & 2.48 & 0.57 & 10.08 & 21.96 & 1.66 & 0.21 & 1.65 & 15.25 & 0.82 & 1.04 \\
\hline & CD at $1 \%$ & 3.59 & 3.35 & 0.76 & 13.44 & 29.60 & 2.24 & 0.28 & 2.22 & 20.55 & 1.11 & 1.40 \\
\hline
\end{tabular}


Similar reports were obtained by Dubey and Maurya (2007) and Suganthi (2008) in bottle gourd, Sarkar and Sirohi (2010) in cucumber, Podder (2010) in snake gourd, Vegad et al., (2011) in bottle gourd, Alli Rani (2013) in ridge gourd, Singh et al., (2013)

The lines DWD local, ERG-2, ERG-1 and DMRG-1 were identified as good general combiners for total fruit yield per hectare in order of merit.The cross DWD local x CO-1, ERG-1 x Deepthi, IC-92637 x Deepthi, HSN local x CO-1 and DMRG-25 x Deepthi were identified as good specific combiners for total fruit yield per hectare in order of merit. Therefore these hybrids can be used commercially by further assessing for its yield stability.

\section{References}

Alli Rani, E. 2013. Genetic studies on growth, yield and mosaic tolerance in ridge Gourd [Luffa actuangula L. (Roxb)]. M.Sc., (Hort.) Thesis, Tamil Nadu Agricultural University, Coimbatore.

Dubey, S.K. and Maurya, I.B. 2007. Combining ability for character related to yield and earliness in Bottle Gourd [Lagenaria siceraria (Mol.) Standl.]. Indian J. Agri. Res., 4 (1): 59-62.

Kempthorne, O. 1957. An Introduction to Genetic Statistics. J. Wiley and Sons, Inc., New York.

Meena, H. S., Kumar, A., Ram, B., Singh, V. V., Meena, P. D., Singh, B. K. and Singh, D. 2015. Combining ability and heterosis for seed yield and its components in Indian Mustard (Brassica juncea L.). J. Agr. Sci. Tech. 17: 1861-1871.

Narasannavar, A.R., Gasti, V. D., Shantappa, T., Mulge, R., Allolli, T. B. and Thammaiah, N. 2014. Heterosis studies in Ridge Gourd [Luffa acutangula (L.) Roxb.] Karnataka J. Agric. Sci., 27 (1): (47-51)

Pali, V and Mehta, N. 2014. Combining ability and heterosis for seed yield and it's attributes in Linseed (Linum usitatissimum L.). The Bioscan. 9 (2): 701-706

Podder, R., Rasul, M. G., Islam, A.K.M.A., Mian, M.A.K. and Ahmed, J. U. 2010. Combining ability and heterosis in Snake Gourd (Tricosanthes cucurminata L). Bangladesh J. Pl. Breed. Genet., 23 (2): 01-06

Sarkar, M. and Sirohi, P.S. 2010. Combining ability analysis for yield and its attributing character in Cucumber. Indian J. Hort., 67 (4): 525-530

Suganthi, M. 2008. LxT analysis in Bottle Gourd [Lagenaria siceraria (Mol.) Stand]. M.Sc. Thesis. Tamil Nadu Agricultural University, Coimbatore.

Vegad, P.M., Vaddoria, M.A., Mehta, D.R. and Naghera, Y.V. 2011. Combining ability analysis in Bottle Gourd. Crop Improvement, 38 (1): 13-17.

Singh, A.K., Pan, R.S. and Bhavana, P. 2013. Heterosis and combining ability analysis in Bitter Gourd (Momordica charantia L.). The Bioscan, 8 (4): 15331536.

\section{How to cite this article:}

Divya S. Kamble, Shivaji Duradundi V.D. Gasti, Shashikanth Evoor, Dileep Kumar A. Masuthi and Sumangala Koulagi. 2018. Combining Ability in Ridge Gourd [Luffa acutangula]. Int.J.Curr.Microbiol.App.Sci. 7(12): 567-577. doi: https://doi.org/10.20546/ijcmas.2018.712.071 